\title{
Revista

\section{Valores de predição da avaliação neurológica e ultra-sonográfica cerebral neonatal em relação ao desenvolvimento de prematuros de muito baixo peso*}

The predictive value of neonatal neurological assessment and neonatal cranial ultrasonography with respect to the development of very low birth weight premature infants

Rosane R. Mello, Maria Virgínia P. D utra, Kátia S. Silva e José Maria A. Lopes Departamento de Neonatologia do Instituto Fernandes Figueira da Fundação O swaldo Cruz (IFF/FIO CRUZ). Rio de Janeiro, RJ - Brasil (R.R.M.); Departamento de Pediatria do IFF/FIO CRUZ. Rio de Janeiro, RJ - Brasil (M.V.P.D., K.S.S.); Departamento de Pesquisa do IFF/FIO CRUZ. Rio de Janeiro, RJ - Brasil (.M.A.L.) 


\title{
Valores de predição da avaliação neurológica e ultra-sonográfica cerebral neonatal em relação ao desenvolvimento de prematuros de muito baixo peso*
}

\section{The predictive value of neonatal neurological assessment and neonatal cranial ultrasonography with respect to the development of very low birth weight premature infants}

\author{
Rosane R. Mello, Maria Virgínia P. D utra, Kátia S. Silva e José Maria A. Lopes \\ Departamento de Neonatologia do Instituto Fernandes Figueira da Fundação O swaldo Cruz (IFF/ \\ FIO CRUZ). Rio de Janeiro, RJ - Brasil (R.R.M.); Departamento de Pediatria do IFF/FIO CRUZ. \\ Rio de Janeiro, RJ - Brasil (M.V.P.D., K.S.S.); D epartamento de Pesquisa do IFF/FIO CRUZ. \\ Rio de Janeiro, RJ - Brasil (.M.A.L.)
}

\begin{abstract}
Resumo
Objetivo Verificar os valores de predição de dois exames (neurológico e ultrasonográfico) realizados no período neonatal, em relação ao desenvolvimento neuromotor e cognitivo de prematuros.

Método Foram estudados 70 prematuros com peso de nascimento inferior a $1.500 \mathrm{~g}$. A média do peso de nascimento e idade gestacional foram, respectivamente, 1.185g (DP:180) e 32,2 semanas (DP:1,4). Foi realizada avaliação neurológica pelo método de Dubowitz e Dubowitz e ultra-sonografia cerebral por volta de 40 semanas de idade corrigida. Após a alta do berçário estas crianças foram acompanhadas no ambulatório de seguimento.
\end{abstract}

Resultados Com idade média de 21 meses (DP: 4,9) corrigidos para a prematuridade, $25,7 \%$ das crianças apresentaram comprometimento neuromotor, e $20,3 \%$, alteração cognitiva. O exame neurológico neonatal foi mais sensível que específico em relação à alteração neuromotora (sensibilidade:77,7\%, especificidade:57,6\%), e cognitiva (sensibilidade: $78,5 \%$, especificidade: $56,4 \%)$, e apresentou baixo valor de predição tanto para alteração neuromotora $(38,9 \%)$ quanto cognitiva $(31,4 \%)$. A ultra-sonografia apresentou elevada especificidade tanto para o desenvolvimento neuromotor $(92,3 \%)$ quanto cognitivo $(89,1 \%)$. O valor preditivo da ultra-sonografia foi satisfatório para anormalidades neuromotoras $(69,2 \%)$, porém baixo para as alterações cognitivas $(50,0 \%)$.

\footnotetext{
*Parcialmente financiado pelo Conselho Nacional de Desenvolvimento Científico e Tecnológico/CNPq (Processo ${ }^{\circ}$ 501072/91).

Baseado na dissertação de mestrado: "Criança: valores de predição da avaliação neurológica neonatal pelo método de Dubowitz e Dubowitz e da ultrasonografia cerebral em relação ao desenvolvimento de prematuros de muito baixo peso", apresentada ao Instituto Fernandes Figueira/FIOCRUZ. Correspondência para/Correspondence to: Rosane Reis de Mello - Rua Eurico Cruz, 23/301 - Jardim Botânico - 22461-200 Rio de Janeiro, RJ Brasil. E-mail: rosanemello@openlink.com.br

Recebido em 14.4.1997. Reapresentado em 26.1.1998. Aprovado em 18.3.1998.
} 
Conclusão

Abstract
Objective
$\begin{aligned} & \text { To verify the predictive value of two tests (neurological assessment and ultra- } \\ & \text { sonography) performed at the time of discharge, with respect to the neuromotor } \\ & \text { and cognitive development of premature infants. }\end{aligned}$

Methods

Results

Conclusion

A associação dos dois exames com resultados anormais apresentou valores de predição mais elevados para as anormalidades nas duas áreas do desenvolvimento.

Prematuro, crescimento e desenvolvimento. Muito baixo peso ao nascer. Exame neurológico. Sintomas cognitivos.

Seventy very low birth weight premature infants (below 1,500g), admitted to the Neonatal Department between 1992 and 1994 were studied. The average birth weight and gestation age were, respectively, 1,185g (SD:180g) and 32.2 weeks (SD: 1.4). At the term correct age, neurological assessment by the Dubowitz \& Dubowitz method and cere bral ultrasonography were carried out. After discharge, the development was monitored at the follow up clinic.

\begin{abstract}
At 21 months correct age (SD: 4.9), neuromotor and cognitive abnormalities were observed, respectively, in $25.7 \%$ and $20.3 \%$ of the infants evaluated. The neonatal neurological assessment was found to be more sensitive than those specific for neuromotor (sensibility:77.7\%, specificity:57.6\%) and cognitive (sensibility: $78.5 \%$, specificity:56.4\%) abnormalities, and exhibited low predictive value for both neumotor and cognitive abnormality. Ultrasonog raphy exhibited high specificity with respect to both neuromotor and cognitive development. The cerebral ultrasonography has also a reasonable predictive value for neuromotor abnormality.
\end{abstract}

The combination of both tests yielded higher predictive values.

Infant, premature. Infant, very low birth weight. Neurologic examination. Cognitive symptons.

\section{INTRO DUÇÃO}

Na década de 90, estudos mostram taxas de sobrevida superiores a $90 \%$ para os prematuros com peso de nascimento acima de $750 \mathrm{~g}^{21}$. Estes bebês são freqüentemente expostos a condições adversas que podem comprometer seu sistema nervoso central e ter como repercussão, alterações do desenvolvimento ${ }^{2}$. Embora grande parte tenha um prognóstico favorável, um número significativo apresenta seqüelas, muitas vezes incapacitantes, com ônus familiar e social importantes. Bennett ${ }^{6}$ relatou prevalência de paralisia cerebral variando de 6 a $10 \%$. Ornstein e col..$^{20}$, em uma revisão dos trabalhos publicados na última década sobre o acompanhamento de prematuros, encontraram taxas de retardo mental oscilando entre 7 e $27 \%$, comprometimento visual de 2 a $38 \%$, perda auditiva de 2 a $44 \%$.

Uma das principais finalidades do acom- panhamento dos bebês prematuros, de muito baixo peso, é a identificação precoce de sinais de anormalidades do desenvolvimento para que uma intervenção apropriada possa ter início, na tentativa de minimizar estas alterações e integrar essas crianças no seio da sociedade.

A hipótese do presente estudo é que a associação dos resultados de exames que avaliam a função cerebral (avaliação neurológica) e a estrutura cerebral (ultra-sonografia) teria maior valor na predição do desenvolvimento futuro de prematuros, do que quando utilizados isoladamente. O objetivo geral do presente trabalho foi verificar os valores de predição de avaliações neonatais realizadas através do exame neurológico pelo método de Dubowitz e Dubowitz ${ }^{12}$ e da ultra-sonografia cerebral, isolados e associados, em relação ao desenvolvimento neuromotor e cognitivo de prematuros com peso de nascimento inferior a $1.500 \mathrm{~g}$, após o primeiro ano de vida. 


\section{MÉTO D O}

Foi analisada a validade diagnóstica da avaliação neurológica neonatal pelo método de Dubowitz e Dubowitz $^{12}$ e da ultra-sonografia cerebral neonatal, tendo como padrão ouro o desenvolvimento neuromotor e cognitivo de prematuros com idades entre 12 e 30 meses, corrigidas para a prematuridade. A correção para a prematuridade é feita subtraindo-se da idade cronológica o número de semanas que faltavam ao nascer para o bebê alcançar o termo (40 semanas). (Idade corrigida $=$ idade cronológica - (40 semanas - idade gestacional ao nascimento, em semanas).

A população estudada compreendeu uma coorte de prematuros com peso de nascimento inferior a $1.500 \mathrm{~g}$, admitidos no Departamento de Neonatologia do Instituto Fernandes Figueira (RJ), entre 1/2/1992 e 31/1/ 1994, e acompanhados por mais de 12 meses de idade corrigida no Ambulatório de Seguimento de Recém-Nascidos de Risco. O critério de prematuridade foi baseado na idade gestacional inferior a 37 semanas (estimada pelo método de Capurro $^{9}$ ). Foram excluídos do estudo os neonatos portadores de síndromes genéticas, de malformações e infecções congênitas, aqueles que foram transferidos para outros serviços e os prematuros oriundos de outros serviços que na admissão tinham idade superior a 40 semanas corrigida para a prematuridade.

\section{Variáveis de Estudo}

Os exames realizados constituíram as variáveis de estudo:

1) Variáveis relacionadas ao período neonatal:

a) Avaliação neurológica pelo método de Dubowitz e Dubowitz ${ }^{12}$; classificação: normal, limítrofe e anormal;

b) ultra-sonografia cerebral; classificação: normal e anormal.

2) Variáveis relacionadas ao desenvolvimento entre 12 e 30 meses de idade corrigida:

a) Avaliação neuromotora: pelo método de Amiel Tison e Grenier ${ }^{3}$ e observação da aquisição dos marcos motores do desenvolvimento; classificação: normal e anormal.

b) Avaliação cognitiva: Escala de Bayley ${ }^{5}$; classificação: normal e anormal.

\section{Variáveis Relacionadas ao Período Neonatal}

\section{Avaliação Neurológica Neonatal pelo Método de Dubowitz e Dubowitz}

O exame foi aplicado por uma médica pediatra quando o bebê alcançou idade corrigida correspondente ao termo (40 semanas). Este exame compreendeu: a observação dos estados da consciência; da capacidade do bebê se habituar a estímulos luminosos e sonoros repetidos; avaliação do tono muscular; observação dos movimentos espontâneos do corpo, pesquisa de reflexos primitivos e profundos; observação de movimentos oculares anormais; orientação auditiva e visual; atenção aos estímulos visuais e auditivos. De acordo com os resultados encontrados, as crianças foram classificadas como em Dubowitz e col ${ }^{13}$ : Normais: se apresentassem tono apropriado para a idade gestacional e não mais do que um sinal anormal. Limítrofes: se apresentassem dois sinais anormais. Anormais: se apresentassem 3 ou mais sinais anormais ou acentuada hipotonia cervical e de tronco. Os sinais anormais considerados foram: inadequado controle da cabeça, tremores e sobressaltos aumentados, polegares em adução persistente, reflexo de Moro anormal, movimentos oculares anormais, inadequada orientação visual ou auditiva, irritabilidade, assimetria e hipertonia da musculatura extensora cervical.

\section{Ultra-sonografia Cerebral}

Foi realizada em idade correspondente a 40 semanas ou próximo à alta do berçário utilizando-se transdutor setorial de $5 \mathrm{MHz}$. Os resultados foram classificados como sendo: normais quando na ausência de anormalidades ultra-sonográficas; e anormais quando os exames mostravam evidências de hemorragia parenquimatosa, leucomalácia periventricular, porencefalia e ventriculomegalia.

\section{Variáveis Relacionadas ao D esenvolvimento entre 12 e 30 Meses de Idade Corrigida}

\section{Avaliação Neuromotora e Cognitiva}

Após a alta do berçário, o acompanhamento dos bebês foi feito no Ambulatório de Seguimento. O diagnóstico neuromotor foi baseado na aquisição dos marcos motores do desenvolvimento e no exame neurológico ${ }^{3}$. A partir do diagnóstico, as crianças foram classificadas segundo Bozinski e $\operatorname{col}^{7,8} \mathrm{em}$ : Normais: quando apresentavam função motora normal, ou seja, andaram até os 18 meses de idade corrigida; sentaram até os 12 meses de idade corrigida e apresentaram exame neurológico normal. Anormais: a) alteração neuromotora menor: se andaram entre 18 e 24 meses de idade corrigida; se apresentaram hipertonia de membros inferiores e hiperreflexia; se andaram ou se mantinham na postura ereta na ponta dos pés persistentemente; se apresentaram hipotonia e leve a moderado atraso motor. b) paralisia cerebral: se não andaram até os 24 meses de idade corrigida ou não sentaram aos 12 meses de idade corrigida; se apresentaram múltiplas anormalidades neurológicas como hiperreflexia e grave atraso motor.

O desenvolvimento cognitivo foi avaliado através do índice de desenvolvimento mental obtido na aplicação da Escala de Bayley de desenvolvimento infantil ${ }^{5}$ por uma psicóloga clínica. De acordo com os escores obtidos, o índice de desenvolvimento mental foi considerado: Normal: escores superiores a 84. Anomal: escores iguais ou inferiores a 84 .

\section{Análise dos Dados}

Foi utilizado para o armazenamento e análise dos dados o programa Epi Info versão 6.02 (1994). Na primeira etapa foram comparadas várias características da população participante do estudo com a população de perdas (para 
verificar a existência de seletividade que poderia prejudicar a validade do estudo). As características contínuas foram medidas através da análise da variância utilizando o teste $\mathbf{F}$ que é equivalente ao teste $\mathbf{t}$ de Student. As diferenças entre porporções foram testadas pelo qui-quadrado. O nível de significância estatística considerado para essas comparações foi de $5 \%(\mathrm{p}<0,05)$. Foram calculadas as prevalências das alterações neuromotoras e cognitivas da população em estudo entre 12 e 30 meses de idade corrigida. Foram calculados os valores de sensibilidade, especificidade ${ }^{16}$ e valores preditivos e os respectivos Intervalos de Confiança do exame neurológico neonatal e da ultrasonografia, isolados e associados e comparados ao padrão-ouro que foi o desenvolvimento neuromotor e cognitivo entre 12 e 30 meses de idade corrigida.

Os dados sociais, maternos e neonatais da população estudada, tiveram sua distribuição descrita através de tabelas de freqüência.

\section{RESU LTAD O S}

Foram admitidos 193 recém-nascidos (RN) com peso de nascimento (PN) inferior a $1.500 \mathrm{~g}$. Deste total, 50 foram a óbito. Entre os 143 sobreviventes, 14 foram excluídos do estudo (um RN a termo, quatro RN com malformação/síndrome genética, cinco bebês admitidos provenientes de outros serviços, com idade gestacional corrigida superior a 40 semanas na internação, quatro RN transferidos para outros serviços). Entre os 129 prematuros com $\mathrm{PN}<1.500 \mathrm{~g}$ que preencheram os critérios de inclusão no estudo, houve perda de 59 crianças $(45,7 \%)$ por falha no cumprimento do protocolo proposto. Dentre os principais motivos estão: 1) não realização de exames: avaliação neurológica neonatal, ultra-sonografia cerebral ou escala de Bayley de desenvolvimento; 2) faltas às consultas agendadas no ambulatório de seguimento; 3) abandono do acompanhamento antes de 12 meses de idade corrigida; 4) óbito no primeiro ano de vida.

As 70 crianças que cumpriram todas as etapas do protocolo proposto foram as que constituíram a população participante do presente estudo.

Diante de um significativo número de perdas do estudo, a preocupação inicial foi comparar as características dessas crianças com as que participaram do estudo (Tabela 1), para verificar se estaria havendo no acompanhamento uma seleção de pacientes mais graves ou menos graves. Foram realizadas várias comparações de médias e proporções entre as características maternas, sociais e do RN. Observouse que os dois grupos foram semelhantes em relação ao peso de nascimento, idade gestacional, sexo, escore de Apgar e patologias neonatais. As diferenças foram em relação à hipertensão arterial materna e sofrimento fetal sendo, nestas duas características, mais acometida a população participante do estudo. Tais informações são apresentadas para que se possa refletir na direção de possível viés de seleção.

Tabela 1 - Dados comparativos entre os prematuros admitidos no estudo e as perdas do estudo, Instituito Fernandes Figueira, RJ, 1992- 1994.

\begin{tabular}{|c|c|c|c|c|c|c|c|c|c|c|c|}
\hline \multirow[b]{2}{*}{ Característica } & \multicolumn{5}{|c|}{ Estudo } & \multicolumn{6}{|c|}{ Perdas } \\
\hline & $\begin{array}{c}\text { Valor } \\
\text { min. }\end{array}$ & $\begin{array}{l}\text { Valor } \\
\text { máx. }\end{array}$ & Média & $\begin{array}{l}\text { Desvio } \\
\text { padrão }\end{array}$ & $\%$ & $\begin{array}{c}\text { Valor } \\
\text { min. }\end{array}$ & $\begin{array}{l}\text { Valor } \\
\text { máx. }\end{array}$ & Média & $\begin{array}{l}\text { Desvio } \\
\text { padrão }\end{array}$ & $\%$ & $p$ \\
\hline Peso nasc.(g) & 640 & 1.490 & 1.185 & 180,0 & & 810 & 1.480 & 1.221 & 185,1 & & 0,143 \\
\hline Capurro (dias) & 204 & 255 & 226 & 10,7 & & 203 & 255 & 225,7 & 11,1 & & 0,896 \\
\hline Apgar $1 \cong$ min. & 1,0 & 10,0 & 5,4 & 2,4 & & 1,0 & 9,0 & 5,4 & 2,4 & & 0,441 \\
\hline Apgar 5ㅇmin. & 3,0 & 10,0 & 7,8 & 1,5 & & 3,0 & 8,0 & 7,8 & 1,5 & & 0,801 \\
\hline $\begin{array}{l}\text { Renda familiar } \\
\text { (sal. mínimo) }\end{array}$ & 0 & 16 & 4,3 & 2,9 & & 1,0 & 20,0 & 4,3 & 3,7 & & 0,998 \\
\hline Sexo masc. & & & & & 34,3 & & & & & 47,5 & 0,128 \\
\hline PIG & & & & & 40,0 & & & & & 32,2 & 0,359 \\
\hline Respirador mecânico & & & & & 38,6 & & & & & 39,6 & 0,900 \\
\hline Membrana hialina & & & & & 31,4 & & & & & 32,7 & 0,877 \\
\hline Apnéia & & & & & 55,8 & & & & & 44,4 & 0,538 \\
\hline Sepsis & & & & & 51,4 & & & & & 35,7 & 0,077 \\
\hline Hipoglicemia & & & & & 31,4 & & & & & 21,8 & 0,230 \\
\hline Hemorragia cerebral & & & & & 8,6 & & & & & 14,5 & 0,293 \\
\hline Hipert.arterial materna & & & & & 64,3 & & & & & 43,7 & 0,027 \\
\hline Sofrim. Fetal & & & & & 62,1 & & & & & 41,8 & 0,038 \\
\hline Escolar.materna $>8$ anos & & & & & 38,8 & & & & & 31,7 & 0,456 \\
\hline
\end{tabular}

$\%$ calculados sobre 0 total de crianças no estudo $(n=70)$ e nas perdas $(n=59)$. 


\section{Características da População Participante do Estudo}

A renda familiar média encontrada foi 4,3 (DP: 2,9) salários-mínimos. Hipertensão arterial acometeu 64,3\% das mães dos bebês, sendo em $23 \%$ dos casos registrados como específicos da gravidez; sofrimento fetal foi detectado em $62,1 \%$ das pacientes; e rotura prolongada de membranas amnióticas em $27,1 \%$ dos casos.

Em relação às características do $\mathrm{RN}$, houve predominância do sexo feminino $(65,7 \%)$ sobre o masculino $(34,3 \%)$. Quanto ao peso do nascimento, $17,1 \%$ dos bebês pesou menos de $1.000 \mathrm{~g}$. A média do peso ao nascer foi $1.185 \mathrm{~g}$ (DP:180g) e da idade gestacional 32,2 semanas (DP:1,4). Em 40\% dos casos, os RN foram pequenos para a idade gestacional (PIG) ${ }^{4}$. A distribuição dos RN de acordo com as patologias e intervenções terapêuticas no período neonatal, é mostrada na Tabela 1 , onde pode-se observar que cerca de 39\% da população estudada necessitou de assistência ventilatória, traduzindo a gravidade do quadro respiratório no início da vida desses bebês. O tempo médio de internação no Departamento de Neonatologia foi 47,6 dias (DP:14,7).

\section{Avaliação N eonatal}

Avaliação Neurológ ica pelo Método de Dubowitze Dubowitz- As 70 crianças receberam esta avaliação com idade média de 40 semanas corrigida para a prematuridade (DP:1,4). Em 34 crianças $(48,6 \%)$ o exame foi considerado normal, em 14 crianças $(20 \%)$, anormal, e em 22 bebês (31,4\%), considerado limítrofe.

Avaliação Ultra-Sonográfica Cerebral - A média da idade corrigida por acasião da realização deste exame foi de 39,6 semanas (DP: 1,9). Em 57 casos $(81,4 \%)$, a ultra-sonografia foi considerada normal e em 13 casos $(18,6 \%)$, anormal. A dilatação ventricular ocorreu em 6 casos $(8,6 \%)$; leucomalácia periventicular em 6 pacientes $(8,6 \%)$ e porencefalia em 3 bebês $(4,3 \%)$. Entre as crianças que desenvolveram dilatação ventricular, em dois casos esta lesão estava associada, um, à leucomalácia e outro, à porencefalia.

\section{Avaliação Após os $\mathbf{1 2}$ Meses de Idade Corrigida}

Desenvolvimento Neuromotor - A média das idades, corrigida para a prematuridade por ocasião da avaliação foi 21,5 meses (DP:4,9). O desenvolvimento neuromotor foi normal em 52 crianças $(74,3 \%)$ e anormal em 18 crianças $(25,7 \%)$ (Tabela 2$)$. Entre estas, seis $(8,6 \%)$ apresentaram paralisia cerebral (uma criança com tetraplegia; três com diplegia e duas com hemiplegia). As outras doze crianças $(17,1 \%)$ apresentaram alterações menos graves.

Tabela 2 - Sensibilidade, especificidade e valores preditivos da avaliação neurológica pelo método de Dubowitz e Dubowitz e ultra-sonografia, isolados e associados em relação ao desenvolvimento neuromotor após os 12 meses de idade corrigida dos prematuros admitidos no Instituto Fernandes Figueira, RJ, 1992-1994.

\begin{tabular}{|c|c|c|c|c|c|c|}
\hline M étodo & $\begin{array}{l}\text { Desenvolv. } \\
\text { Anormal }\end{array}$ & $\begin{array}{l}\text { neuromotor } \\
\mathrm{N} \text { ormal }\end{array}$ & $\begin{array}{c}\text { Sensibilidade } \\
(\%) \\
(\mathrm{IC}) \\
\end{array}$ & $\begin{array}{c}\text { Especificidade } \\
(\%) \\
(\mathrm{IC}) \\
\end{array}$ & $\begin{array}{c}\text { Valor pred. }+ \\
(\%) \\
(\text { IC) }\end{array}$ & $\begin{array}{c}\text { Valor predit. - } \\
(\%) \\
(\mathrm{IC})\end{array}$ \\
\hline \multicolumn{7}{|l|}{ Dubowitz } \\
\hline Normal & 14 & 22 & 77,7 & 57,6 & 38,9 & 88,2 \\
\hline Anormal & 4 & 30 & $(51,9-92,6)$ & $(43,3-71,0)$ & $(23,6-56,5)$ & $(71,6-96,2)$ \\
\hline \multicolumn{7}{|l|}{ Ultra-sonografia } \\
\hline Normal & 9 & 4 & 50,0 & 92,3 & 69,2 & 84,2 \\
\hline Anormal & 9 & 48 & $(26,8-73,2)$ & $(80,6-97,5)$ & $(38,9-89,6)$ & $(71,6-92,1)$ \\
\hline \multicolumn{7}{|c|}{ D ubowitz e U Itra-sonografia } \\
\hline Ambos anormais & 8 & 2 & 44,4 & 96,2 & 80,0 & 83,3 \\
\hline Normal p/ 1 método & 10 & 50 & $(22,2-68,7)$ & $(85,7-99,3)$ & $(44,2-96,5)$ & $(71,0-91,3)$ \\
\hline \multicolumn{7}{|c|}{ Dubowitz ou Ultra-sonografia } \\
\hline 1 ou outro anormal & 15 & 24 & 83,3 & 53,8 & 38,5 & 90,3 \\
\hline Ambos normais & 3 & 28 & $(57,7-95,6)$ & $(39,6-67,5)$ & $(23,8-55,2)$ & $(73,1-97,5)$ \\
\hline
\end{tabular}


Desenvolvimento Cognitivo - Das 70 crianças submetidas a este teste, somente uma $(1,4 \%)$ não pôde ter seu escore final quantificado devido à perda visual total bilateral. Conseqüentemente, esta criança não foi incluída nos cálculos que diziam respeito a esta escala.A idade média das crianças, corrigida para a prematuridade por ocasião deste teste foi 21,4 meses (DP:4,9). A média encontrada para o índice de desenvolvimento mental entre as crianças participantes do estudo foi 99,0 (DP:20,9). O índice de desenvolvimento mental foi normal em 55 crianças $(79,7 \%)$ e anormal em 14 crianças $(20,3 \%)$ (Tabela 3). Entre as 14 crianças com índice de desenvolvimento mental anormal, $11(78,6 \%)$ também apresentavam concomitantes alterações neuromotoras: $5 \mathrm{com}$ paralisia cerebral e $6 \mathrm{com}$ alterações de menor gravidade. Somente uma criança com paralisia cerebral obteve índice de desenvolvimento mental dentro dos limites normais. Alterações cognitivas sem concomitância de comprometimento neuromotor foram encontradas em três crianças. Houve concomitância de alterações neuromotoras e cognitivas em $15,7 \%$ das crianças.

\section{Valores de Predição da Avaliação Neurológica e da Ultra-sonografia, em Relação ao D esenvolvimento após os 12 Meses de Idade Corrigida}

Para os cálculos destes valores, optou-se em trabalhar com somente duas categorias de resultados (normal e anormal). Como a classificação dos resultados da avaliação de Dubowitz incluía três categorias, os valores de sensibilidade, especificidade e valores preditivos, foram calculados incluindo as avaliações limítrofes, na categoria anormal.

\section{Em Relação ao D esenvolvimento N euromotor}

A Tabela 2 apresenta os resultados de sensibilidade, especificidade, valores preditivos, e os respectivos Intervalos de Confiança (IC), expressos em percentagem, dos dois exames, isolados e associados, em relação ao desenvolvimento neuromotor. Isoladamente, as 36 avaliações neurológicas neonatais anormais predisseram $39 \%$ das anormalidades após o primeiro ano de vida. Os valores de sensibilidade, especificidade e valores preditivos positivo e negativo da avaliação neurológica neonatal foram, respectivamente, $77,7 \%$, $57,6 \%, 38,9 \%$ e $88,2 \%$, em relação ao desenvolvimento motor.

Os exames ultra-sonográficos anormais no período neonatal predisseram $70 \%$ dos casos de alterações neuromotoras. Os valores de sensibilidade, especificidade e valores preditivos positivo e negativo foram, respectivamente, $50,0 \%, 92,3 \%$, $69,2 \%$ e $84,2 \%$.

Quando os exames (avaliação neurológica neonatal pelo método de Dubowitz e Dubowitz e ultra-sonografia) foram analisados em associação e

Tabela 3 - Sensibilidade, especificidade e valores preditivos da avaliação neurológica pelo método de Dubowitz e Dubowitz e da ultra-sonografia em relação ao desenvolvimento cognitivo após os 12 meses de idade corrigida dos prematuros admitidos no Instituto Fernandes Figueira, RJ, 1992-1994

\begin{tabular}{|c|c|c|c|c|c|c|}
\hline M étodo & $\begin{array}{l}\text { Desenv. } \\
\text { Anormal }\end{array}$ & $\begin{array}{l}\text { gnitivo } \\
\text { Normal }\end{array}$ & $\begin{array}{c}\text { Sensibilidade } \\
(\%) \\
\text { (IC) }\end{array}$ & $\begin{array}{l}\text { Especificidade } \\
\text { (\%) } \\
\text { (IC) }\end{array}$ & $\begin{array}{c}\text { Valor predit. } \\
(\%) \\
\text { (IC) }\end{array}$ & $\begin{array}{c}\text { Valor predit. - } \\
(\%) \\
\text { (IC) }\end{array}$ \\
\hline \multicolumn{7}{|l|}{ Dubowitz } \\
\hline Normal & 3 & 31 & $(48,6-94,3)$ & $(42,4-69,4)$ & $(17,4-49,4)$ & $(75,2-97,7)$ \\
\hline \multicolumn{7}{|l|}{ Ultra-sonografia } \\
\hline Anormal & 6 & 6 & 42,9 & 89,1 & 50,0 & 86,0 \\
\hline Normal & 8 & 49 & $(18,8-70,4)$ & $(77,1-95,5)$ & $(22,3-77,7)$ & $(73,7-93,3)$ \\
\hline \multicolumn{7}{|c|}{ Dubowitz e ultra-sonografia } \\
\hline Ambos anormais & 5 & 4 & 35,7 & 92,7 & 55,6 & 85,0 \\
\hline Normal p/ 1 método & 9 & 51 & $(14,4-64,4)$ & $(81,6-97,6)$ & $(22,7-84,7)$ & $(72,9-92,5)$ \\
\hline \multicolumn{7}{|c|}{ Dubowitz ou ultra-sonografia } \\
\hline 1 ou outro anormal & 12 & 26 & 85,7 & 52,7 & 31,6 & 93,5 \\
\hline Ambos normais & 2 & 29 & $(56,2-97,5)$ & $(38,9-66,1)$ & $(18,0-48,8)$ & $(77,2-98,9)$ \\
\hline
\end{tabular}


ambos foram anormais, notou-se que o poder de predição das alterações neuromotoras subiu para $80 \%$ (Tabela 2). A sensibilidade, especificidade e valores preditivos positivo e negativo dos dois exames associados e anormais em relação ao desenvolvimento motor foram, respectivamente, $44,4 \%, 96,2 \%, 80,0 \%$ e $83,3 \%$.

Entre as 10 crianças que tiveram ambos os exames neonatais anormais, 8 apresentaram comprometimento neuromotor, sendo quatro com paralisia cerebral e quatro com alterações de menor gravidade. As quatro crianças com paralisia cerebral apresentaram concomitante alteração cognitiva. Porém, entre as quatro com comprometimento neuromotor menos grave, só uma obteve índice de desenvolvimento mental anormal. Entre estas crianças com os dois exames neonatais anormais, duas delas não tiveram comprometimento neuromotor após o primeiro ano de vida e, em uma delas, também não foi encontrada alteração cognitiva. A outra criança não pôde ter o escore do índice mental quantificado, devido ao déficit visual.

A mais alta sensibilidade $(83,3 \%)$ foi atingida quando se considerou a anormalidade do exame neurológico neonatal ou da ultra-sonografia.

\section{Em Relação ao D esenvolvimento Cognitivo}

Entre as 14 crianças com avaliação de Dubowitz anormal, $7(53,8 \%)$ apresentaram após o primeiro ano de vida desenvolvimento cognitivo em níveis anormais. Quando agrupamos as avaliações classificadas como limítrofes no grupo das anormais (Tabela 3), cerca de $31 \%$ destas crianças, após os 12 meses, apresentaram alterações cognitivas. Novamente a maior sensibilidade $(85,7 \%)$ foi atingida quando se considerou anormalidades presentes em um ou outro exame neonatal.

Os exames ultra-sonográficos anormais conseguiram predizer $50 \%$ dos casos de déficit cognitivo entre as crianças após os 12 meses. A sensibilidade encontrada foi $42,9 \%$, a especificidade $89,1 \%$ e o valor preditivo negativo $86,0 \%$.

Quando analisados associados os resultados da avaliação neurológica neonatal e os da ultrasonografia cerebral, percebe-se que quando ambos foram anormais, conseguiram predizer $55,6 \%$ dos casos de déficits cognitivos entre as crianças após o primeiro ano de vida. A sensibilidade foi $35,7 \%$, a especificidade foi elevada $(92,7 \%)$ e o valor preditivo negativo $85,0 \%$. Porém, quando foram considerados alterados um ou outro exame realizados próximo à alta do berçário, observou-se aumento na sensibilidade $(85,7 \%)$ destes exames em relação ao diagnóstico de déficit cognitivo após um ano de idade corrigida.

\section{DISCU SSÃO}

Este é um dos primeiros trabalhos nacionais que aborda o desenvolvimento de prematuros de muito baixo peso. Poucos estudos ${ }^{15,18}$ sobre este tema foram publicados no Brasil e é provável que isto seja reflexo das dificuldades encontradas na realização de estudos longitudinais que avaliem resultados a curto, a médio e, principalmente, a longo prazo. As condições socioeconômicas e culturais da população admitida no Departamento de Neonatologia talvez permitam explicar, parcialmente, o grande número de faltas às consultas de acompanhamento, dificultando e impossibilitando o cumprimento de todas as etapas do protocolo proposto. Apesar das dificuldades enfrentadas durante as etapas do trabalho, conseguiuse cumprir todo o protocolo em $54 \%$ da população de prematuros incluídos no estudo e acompanhar por mais de 12 meses, $71,3 \%$ da população dos elegíveis para o trabalho.

Em relação ao desenvolvimento neuromotor, na população estudada, $8,6 \mathrm{~b} \%$ crianças foram portadoras de paralisia cerebral. Escobar e col. ${ }^{14}$ (1991) relataram prevalência de $7,7 \%$ desta anormalidade entre os sobreviventes da coorte de RN de muito baixo peso, em sua meta-análise. PintoMartin e col. ${ }^{22}$ encontraram $14,4 \%$ de portadores de paralisia cerebral em sua população de prematuros e Allen e Capute 1 , 19\%. Portanto, as taxas de anormalidades neuromotoras graves encrontradas estão dentro dos níveis de prevalência descritos na literatura estrangeira. Foram encontrados $17,1 \%$ das crianças com alterações neuromotoras de menor gravidade. Taxas semelhantes (16\%) foram encontradas por Allen e Capute ${ }^{1}$. Vohr e col. ${ }^{23}$ relataram taxas superiores $(28,5 \%)$.

Quanto ao desenvolvimento cognitivo, a maioria das crianças estudadas $(79,7 \%)$ obteve o índice de desenvolvimento mental dentro dos limites normais. Gross e col. ${ }^{17}$ relataram que $70 \%$ das crianças prematuras por eles acompanhadas apresentaram desenvolvimento mental normal.

$\mathrm{Na}$ aplicação do exame neurológico neonatal pelo método de Dubowitz e Dubowitz, foram encontrados 48,6\% dos exames normais, 31,4\% limítrofes e 20\% anormais. Dubowitz e col. ${ }^{13}$ encontraram 53,5\% dos 
exames neurológicos normais; $15,5 \%$ limítrofes e $31 \%$ anormais. Bozinski e col. ${ }^{8}$ encontraram $45 \%$ dos prematuros com resultados normais, $30 \%$ limítrofes e $25 \%$ com resultados anormais. Comparando com os presentes resultados, percebe-se que as taxas de anormalidades encontradas por esses autores foram superiores às observadas. Talvez a média da idade gestacional das crianças por eles avaliadas (30 semanas), inferior à média da idade gestacional da população estudada $(32,2$ semanas, DP:1,4), justifique estas taxas superiores de anormalidades devido ao maior risco de complicações no período neonatal a que estas crianças estão sujeitas.

Quando foram agrupadas as crianças com exame neurológico neonatal limítrofe, junto à categoria dos anormais, percebe-se um grande número de testes falso-positivos e isto, provavelmente, ocorreu em função das alterações transitórias de tono e postura, que são achados freqüentes em crianças de muito baixo peso e que gradualmente vão melhorando para normalizarem entre o primeiro e o segundo anos de vida $^{11}$. Como foi observado, entre os 22 prematuros com exames neonatais limítrofes, 18 normalizaram seu exame neuromotor entre 12 e 30 meses de idade corrigida. Entre as 4 crianças que não normalizaram, duas desenvolveram paralisia cerebral. Torna-se difícil comparar os presentes resultados da avaliação neurológica neonatal com os de outros autores, como Dubowitz e col. ${ }^{13}$, pois esses autores não incluíram as crianças limítrofes nos cálculos de predição do desenvolvimento global, só considerando as crianças com exames neurológicos neonatais normais e anormais. Desta forma, encontraram sensibilidade de $83 \%$, especificidade de $80 \%$, valores preditivo positivo $64 \%$ e negativo $91 \%$. Também foi de difícil comparação com os estudos de Allen e Capute ${ }^{1}$ devido a diferentes critérios metodológicos empregados na classificação dos exames neurológicos normais e anormais. Estes autores encontraram sensibilidade de $70 \%$, especificidade de $77 \%$, valor preditivo positivo de $65 \%$, valor preditivo negativo de $81 \%$ em relação às anormalidades motoras.

Deve-se levar em consideração na nossa prática diária, a finalidade a que se propõe o uso do teste. Se a finalidade é a predição de anormalidade neuromotora futura, necessita-se de um teste com alto valor preditivo positivo. Se se deseja no momento da alta do berçário identificar um grupo de prematuros que necessita de maior vigilância de seu desenvolvimento e de algum tipo de intervenção precoce, precisa-se discutir as propriedades do teste. Portanto, quando se desloca os bebês com resultados limítrofes para o grupo dos anormais, encontra-se sensibilidade de $77 \%$ e com isto aumenta-se o número de crianças com alterações neuromotoras identificadas precocemente. Por outro lado, há algumas com testes falso-positivos. Porém, considera-se preferível incluir essas crianças com testes falso-positivos no grupo que necessitará de maior vigilância e intervenção do que deixar de intervir pois essas crianças poderão vir a apresentar algum comprometimento neuromotor futuro.

No que diz respeito à avaliação de Dubowitz em relação ao desenvolvimento cognitivo, os resultados foram semelhantes aos encontrados no desenvolvimento neuromotor, ou seja, valor preditivo negativo e sensibilidade elevados, e valor preditivo positivo e especificidade baixos. Se a finalidade do teste é identificar um grupo de crianças que possa se beneficiar de assistência para maximizar as habilidades cognitivas, necessita-se de um teste muito sensível. O valor preditivo negativo foi elevado, o que talvez permita tranquilizar os familiares, uma vez que entre as crianças com Dubowitz normal, mais de $90 \%$ delas apresentaram índice de desenvolvimento mental dentro dos limites normais para a idade.

A ultra-sonografia cerebral em relação ao desenvolvimento motor apresentou baixa sensibilidade, identificando $50 \%$ das crianças com anormalidades neuromotoras. Por outro lado, apresentou alta especificidade, revelando que das crianças normais após um ano, mais de $90 \%$ apresentaram ultra-sonografias normais próximo à alta do berçário. O valor preditivo positivo encontrado indicou que em relação às ultrasonografias alteradas, $70 \%$ das crianças desenvolveram alterações neuromotoras. O valor preditivo negativo também pode ser considerado satisfatório, indicando que em relação aos exames ultra-sonográficos normais, quase $85 \%$ das crianças estavam livres de alterações neuromotoras. Autores ${ }^{19,22}$ que estudaram os valores de predição da ultra-sonografia em relação ao desenvolvimento neuromotor relataram baixo valor preditivo positivo $(37,0 \%, 52,4 \%)$ e elevado valor preditivo negativo $(88,0 \%, 95,8 \%)$.

Quando se analisa a ultra-sonografia isoladamente, em relação ao desenvolvimento cognitivo, verificam-se resultados semelhantes aos encontrados para o neuromotor, porém, com valor preditivo positivo mais baixo, indicando que dos exames ultra-sonográficos anormais, $50 \%$ das crianças apresentaram alteração cognitiva. Costello e col. ${ }^{10}$ relataram que a ultra-sonografia cerebral foi 
mais fiel na predição de anormalidades neuromotoras do que cognitivas, quando analisou sua população aos 4 anos de idade.

A associação dos dois exames alterados (neurológico neonatal e ultra-sonografia) aumentou o valor preditivo positivo em relação às anormalidades neuromotoras $(80,0 \%)$, comparado aos valores preditivos dos testes isolados $(38,9 \%$ e $69,2 \%)$.

Também considera-se importante a possibilidade de tranqüilizar os pais dos bebês que apresentaram ambos os exames normais realizados próximo à alta do berçário. Com o valor preditivo negativo superior a $80 \%$, embora não se possa garantir um desenvolvimento motor normal, pode-se utilizar destes dados para acalmar os familiares desses bebês.

Ainda em relação aos exames associados, considera-se importante saber os valores de predição quando um ou o outro exame (neurológico neonatal ou ultrasonografia) estava alterado. Foi encontrada sensibilidade superior a $80 \%$, indicando que mais de $80 \%$ das crianças com anormalidades neuromotoras após um ano de vida foi identificada por um ou o outro exame anormal no período neonatal. $\mathrm{O}$ valor de predição para anormalidades neuromotoras foi baixo $(38,5 \%)$ às custas de grande número de testes falso-positivos. Por outro lado, o valor preditivo negativo foi excelente $(90,3 \%)$.

No que diz respeito aos exames de Dubowitz e ultra-sonografia analisados conjuntamente em relação ao desenvolvimento cognitivo após os 12 meses, percebe-se que quando ambos os exames foram anormais, a sensibilidade foi baixa, indicando que das crianças com alteração cognitiva após um ano, menos de $40 \%$ foi identificada pelos testes no período neonatal, porém a especificidade foi elevada. O valor preditivo positivo $(55,6 \%)$ não foi satisfatório. Por outro lado, o valor preditivo negativo foi bom, indicando que quando os dois testes (Dubowitz e Ultra-sonografia) foram normais, 85\% das crianças após o primeiro ano de vida estavam com o desenvolvimento cognitivo adequado. Se se considerar um ou o outro teste anormal (Dubowitz ou Ultra-sonografia) nota-se que a sensibilidade dos testes foi de $85,7 \%$. Isto permite concluir que $85 \%$ das crianças com alteração cognitiva foi identificada quando um ou o outro teste (Dubowitz e Ultrasonografia) foi anormal. Enquanto o valor preditivo positivo foi baixo, o valor preditivo negativo encontrado foi excelente $(93,5 \%)$, o que ajuda a tranqüilizar as famílias desses bebês que apresentaram ambos os testes normais.

Foi possível concluir que: a) As taxas de anormalidades neuromotoras e cognitivas encontradas na população estudada encontram-se dentro dos níveis de prevalência descritos na literatura estrangeira. b) No Brasil, onde existe pouca disponibilidade de recursos de avançada tecnologia, ao se associar os resultados de dois exames de baixo custo, não invasivos, e de rápida aplicação, está-se aumentando a validade preditiva desses dois exames como testes diagnósticos para futuras anormalidades neuromotoras. Os valores preditivos para anormalidades neuromotoras e cognitivas foram superiores quando os dois testes (avaliação neurológica e ultra-sonográfica) foram analisados e ambos encontraram-se alterados. c) $\mathrm{O}$ valor de predição dos testes associados em relação às alterações neuromotoras pode ser considerado satisfatório, porém não o foi em relação às alterações cognitivas. d) Com a finalidade de identificar aquelas crianças que futuramente apresentarão alterações neuromotoras e se beneficiarão de intervenção, sugere-se que sejam analisados os resultados dos dois exames (neurológico e ultra-sonográfico) antes da alta hospitalar.

\section{AGRADECIMENTOS}

À Prof. Denise S. Morsh pela aplicação da Escala de Bayley de desenvolvimento às crianças do estudo e à Dra. Maria Dalva B. Meio pela colaboração no acompanhamento pediátrico das crianças no ambulatório de seguimento. À Dra. Maura C. Cecherelli pela aplicação da avaliação neurológica neonatal, à Prof. Dra. Alexandra Monteiro pela revisão dos exames ultra-sonográficos e à Prof. Vânia Matos Fonseca pela imprescindível colaboração. 


\section{REFERÊNCIAS}

1. ALLEN, M.C. \& CAPUTE, A.J. Neonatal neurodevelopmental examination as a predictor of neuromotor outcome in premature infants. Pediatrics, 83: 498- 506, 1989.

2. ALLEN, M.C. Assessment of risk for developmental disability. In: Jones, M.D. et al., ed. Hospital care of the recovering NICU infant. Baltimore, Williams \& Wilkins, 1991. p.149-60.

3. AMIEL TISON, C. \& GRENIER, A. Neurological assessment during the first year of life. New York, Oxford, University Press, 1986.

4. B ATTAGLiA, F.C. \& LUBCHENCO, L.O. A pratical classification of newborn infants by weight and gestational age. J. Pediatr., 71:159-63, 1967.

5. B AYLEY, N. Bayley scales of infant development. New York, Psychological Corporation, 1969.

6. BENNETT, F.C. Developmental outcome. In: Avery, G.B.; Fletcher, M.A.; Mac Donald, M.G. ed. Neonatologypathophysiology and management of the newborn. 4th. ed Philadelphia, J.B. Lippincott Company, 1994. p.1367-86.

7. BOZYNSKI, M.E.A. et al. Cranial ultrasonography and the prediction of cerebral palsy in infants weighting $\leq 1200$ grams at birth. Dev. Med. Child Neurol., 30: 342-8, 1988.

8. BOZYNSKI, M.E.A. et al. Cranial ultrasonography and neurologic examination of extremely preterm infants. Dev. Med. Child Neurol., 32: 575-81, 1990.

9. CAPURRO, H. et al. A simplified method for diagnosis of gestational age in the newborn infant. J. Pediatr., 93: $120-2,1978$.

10. COSTELLO, A.M.L. et al. Prediction of neurodevelopmental impairment at four years from brain ultrasound appearance of very preterm infants. Dev. Med. Child Neurol., 30: 711-22, 1988.

11. D'EUGENIO, D.B .et al. Developmental outcome of preterm infants with transient neuromotor abnormalities. AJDC., 147: 570-4, 1993.
12. DUBOWITZ, L.M.S.\& DUBOWITZ, V. The neurological assessment of the preterm and full term newborn infant. London, Spastics International Medical Publications, 1981. (Clinics in Developmental Medicine, 79).

13. DUBOWITZ, L.M.S. et al. Correlation of neurologic assessment and outcome at one year.J. Pediatr., 105: 452-6, 1984.

14. ESCOBAR, G.J.et al. Outcome among surviving very low birth weight infants: a meta-analysis. Arch. Dis. Child., 66: 204- 11, 1991.

15. FIGUEIREDO, E.M. et al. Bebê de alto risco: abordagem fisioterápica e terapêutica ocupacional. Infanto, 3: 8-11, 1995.

16. LETCHER, R.H. et al. Diagnóstico. In: Fletcher, R.H. et al. Epidemiologia clínica: bases científicas da conduta médica. 2 ed. rev. Porto Alegre, Artes Médicas, 1991. p. 68-106.

17. GROSS, S.S.et al. Impact of a matched term control group on interpretation of developmental performance in preterm infants. Pediatrics, 90: 681-7, 1992.

18. MEIO, M.D.B. et al. Utilização do método de Gesell na avaliação do desenvolvimento de crianças que necessitaram de ter apia intensiva neonatal. J. Pediatr., 68: 18-20, 1992.

19. NG, P.C. \& DEAR, P.R.F. The predictive value of a normal ultrasound scan in the preterm baby -a meta-analysis. Acta Paediatr. Scand., 79: 286-91, 1990.

20. ORNSTEIN, M. et al. Neonatal follow up of very low birthweigth/ extremely low birthweigth infants to school age: a critical overview. Acta Paediatr. Scand., 80: 741-8, 1991.

21. PHILLIP, A.G.S. Neonatal mortality rate: is further improvement possible?. J. Pediatr., 126:427-33, 1995.

22. PINTO-MARTIN, J.A.et al. Cranial ultrasound prediction of disabling and nondisabling cerebral palsy at age two in a low birth weight population. Pediatrics., 95: 249-54, 1995.

23. VOHR, B.R. \& COLL, C.T.G. Neurodevelopmental and school performance of very birth weight infants: a seven year longitudinal study. Pediatrics, 76: 345-50, 1985. 